\title{
Incidence of and risk factors for secondary ocular hypertension in moderate to severe infectious ulcerative keratitis
}

This article was published in the following Dove Press journal:

Clinical Ophthalmology

\section{Darin Sakiyalak \\ Yuwared Chattagoon}

Department of Ophthalmology, Faculty of Medicine Siriraj Hospital, Mahidol University, Bangkok, Thailand
Purpose: The aim of this study was to investigate the incidence of and risk factors for secondary ocular hypertension (OHT) in patients with moderate to severe infectious ulcerative keratitis and to evaluate the long-term outcome of patients with secondary OHT.

Patients and methods: A retrospective chart review of 346 patients with moderate to severe infectious keratitis admitted to Siriraj Hospital during the period from 1 January 2005 to 31 May 2008 was conducted. Secondary OHT was defined as intraocular pressure (IOP) greater than $21 \mathrm{mmHg}$ or a consistent demonstration of higher IOP in the affected eye of $8 \mathrm{mmHg}$ or greater, at any time before resolution of the ulcer. The incidence of and the risk factors for secondary OHT were determined. Among the patients with secondary OHT, the incidence of and the risk factors for persistent elevated IOP and blindness at the last follow-up were also evaluated.

Results: Two hundred and two eyes were included in the study. The incidence of secondary OHT was $45.5 \%$. Severe keratitis and severe anterior chamber cells' reaction were the risk factors for IOP elevation during active keratitis ( $P=0.003$ and 0.018 , respectively). Long-term data were available for 69 patients with OHT; 32.9\% (22/69) developed persistent IOP elevation after keratitis resolved. Older age $(P=0.007)$ and hyperosmotic agents used during active keratitis $(P=0.028)$ were associated with persistent IOP elevation. Age was also associated with blindness among the patients with secondary OHT $(P=0.002)$.

Conclusion: Moderate to severe infectious keratitis was associated with a high incidence of secondary OHT. Two main risk factors were severe corneal infiltration and severe intraocular inflammation. One-third of the patients with OHT developed persistent elevated IOP after keratitis was healed. Older age and hyperosmotic agents used during active keratitis were significantly associated with persistent elevated IOP. Older age was also associated with poorer visual outcome.

Keywords: intraocular pressure, glaucoma, keratitis, corneal ulcer

\section{Introduction}

Infectious ulcerative keratitis is a significant disease of the cornea among Asian populations. Complications of infectious keratitis, such as corneal scarring, corneal melting perforation, endophthalmitis, panophthalmitis, and secondary glaucoma, can result in blindness. According to our review of the literature, only one study has been conducted on the incidence of and risk factors for developing ocular hypertension (OHT) in infectious keratitis. Zarei-Ghanavati et al found elevation of intraocular pressure (IOP) during active microbial keratitis to be significantly associated with the size of ulcer, presence of diabetes mellitus, and prior ocular surgery. ${ }^{1}$ That study was conducted in patients with culture-proven microbial keratitis at all levels of
Correspondence: Darin Sakiyalak Medicine Siriraj Hospital, Mahidol University, 2 Wanglang Road Bangkoknoi, Bangkok 10700, Thailand Tel +6624198037

Fax +6624141232

Email dsakiyalak@gmail.com 
severity. Small ulcer (ulcer size $<2 \mathrm{~mm}$ ) can generally be managed on an outpatient basis with good outcome and less complications. Seventeen percent of small-sized ulcer was associated with OHT, as compared to $32 \%$ of moderate and large ulcers in that study. Moderate and large ulcers are more difficult to manage and may have more risk of complications, including the development of secondary OHT, which may result in new and more serious problems, including chronic glaucoma and vision loss. However, the problems and risk factors associated with moderate and severe infectious keratitis are not well established. Understanding the risk of OHT development along with an accurate diagnosis and appropriate treatment will improve patient outcomes in moderate and high levels of infection. Accordingly, the aim of this study was to investigate the incidence of and risk factors for secondary OHT in patients with moderate to severe infectious ulcerative keratitis. Furthermore, the long-term outcome of patients with secondary OHT was evaluated.

\section{Patients and methods}

This retrospective chart review was conducted in patients with moderate or severe infectious ulcerative keratitis who were treated as inpatients at the Department of Ophthalmology, Siriraj Hospital during the study period from 1 January 2005 to 31 May 2008. Siriraj Hospital is Thailand's largest university-based national tertiary referral center. The protocol for this study was approved by the Siriraj Institutional Review Board, Faculty of Medicine Siriraj Hospital, Mahidol University, Bangkok, Thailand (approval no 188/2553). Patient consent was waived by the Siriraj Institutional Review Board as the retrospective study posed no risk to the patients, and data confidentiality was maintained at all times.

Patients meeting any one of the following criteria were excluded: pre-existing glaucoma or OHT in the study eye, OHT or primary glaucoma in the fellow eye, history of intraocular surgery other than uncomplicated cataract surgery, or patients with incomplete medical records. Patients who presented with corneal perforation, underlying viral keratitis, or ophthalmic conditions requiring prolonged use of steroids were also excluded.

Diagnosis of infectious keratitis was based on clinical diagnosis with severity grading based on modified Jones grading criteria. ${ }^{2}$ Moderate infectious keratitis is characterized by corneal lesion size $2-6 \mathrm{~mm}$ in diameter, involving one-third to two-thirds of depth, with moderate to severe anterior segment inflammation. Severe infectious keratitis is associated with corneal lesion larger than $6 \mathrm{~mm}$ in diameter, extending to the inner third of the cornea, with severe anterior segment inflammation or hypopyon.

On admission, all patients underwent comprehensive eye examination, including visual acuity (VA) test, slit-lamp biomicroscopy, IOP measurement, and fundus examination, followed by corneal scrapings. Corneal specimen was prepared for Gram stain, 10\% KOH preparation, and microbiological culture. Routine cultures for corneal specimens during the study period included bacterial, fungal, and acanthamoebic media. If the results of initial cultures were negative or the clinical conditions worsened, corneal scrapings would be repeated. Corneal biopsy was considered when cultures remained negative and no clinical response was observed.

OHT was defined as an IOP of greater than $21 \mathrm{mmHg}$ as well as a consistent demonstration of higher IOP in the affected eye of $8 \mathrm{mmHg}$ or greater, as measured by Tono-Pen (Reichert Tono-Pen ${ }^{\circledR}$ XL applanation tonometer; Reichert, Inc., NY, USA) at any time before resolution of the ulcer. Anterior chamber (AC) cell reaction was classified based on the Standardization of Uveitis Nomenclature criteria ${ }^{3}$ as follows: mild (grade $0-1+$ ), moderate (grade $2+$ to $3+$ ), and severe (grade 4+ or hypopyon).

Data that were collected and analyzed included: age, gender, underlying systemic and ocular diseases, prior ocular surgery, clinical presentation, presenting VA, IOP, other complications of infectious keratitis, treatment for infectious keratitis and complications, microbiological evaluation results, and length of stay. Long-term clinical data including duration of follow-up, presenting VA, IOP, antiglaucoma medications, and further glaucoma surgical procedures were also collected and analyzed. The data were collected by chart review. The patients who were on glaucoma medications but had followed up $<1$ month after discharge were excluded from the long-term analysis.

\section{Statistical analysis}

Data analysis was performed using PASW Statistics version 18.0 (SPSS, Inc., Chicago, IL, USA). Categorical variables were expressed using number and percentage, whereas continuous variables were described as mean \pm SD or median and range, as appropriate. Pearson's chi-squared test or Fisher's exact test was used to compare proportions of categorical variables between groups. Mann-Whitney $U$ test was used to compare continuous variables. Simple and multiple logistic regression analyses were used to identify factors associated with OHT in corneal ulcer, persistent elevation of IOP, and poor visual outcome at the last follow-up. OR was used to measure factors associated with $\mathrm{OHT}$ in corneal ulcer. 
All tests of significance were two tailed, and $P$-values $<0.05$ were considered statistically significant.

\section{Results}

Of 346 infectious ulcerative keratitis patients who were reviewed, 202 patients met the inclusion criteria and were enrolled. The overall mean age of patients was $42.6 \pm 19.4$ years (range: 1-84). Ninety-five cases (47\%) were male and 107 cases $(53 \%)$ were female. One hundred forty-two patients $(70.3 \%)$ presented with severe infectious keratitis and 60 patients $(29.7 \%)$ presented with moderate keratitis. IOP elevation was found in 92 patients $(45.5 \%)$. Demographic and clinical characteristics of patients in the OHT group and control group are presented in Table 1. In 86 eyes (42.6\%), the pathogenic organisms were able to be identified by culture or corneal biopsy, $39.2 \%$ by culture, and $3.4 \%$ by corneal biopsy. In other 116 eyes $(57.4 \%)$, the organisms were identified by clinical presentation and clinical response to treatment. Leading pathogenic organisms were bacteria (56.9\%), followed by fungi (27.7\%), mixed infection (3.0\%), and Acanthamoeba (2.0\%). The most common isolated organism was Pseudomonas aeruginosa, found in 38 cases (Table 2). In seven patients with bacterial infection, more than one organism was identified. Among six patients with mixed infection, four patients had bacteria and fungi isolated and two patients had bacteria and Acanthamoeba isolated. In 21 patients $(10.4 \%)$, the organism could not be isolated, but keratitis was controlled with a combination of antibiotics and antifungal medications, and they were classified as unidentified. Incidence of secondary OHT caused by different types of organisms and severity of infection are presented in Table 3.

Simple logistic regression and multiple logistic regression analyses were performed to assess the relationships

Table I Demographic and clinical data of OHT group and control group

\begin{tabular}{|c|c|c|c|}
\hline Demographics & OHT group & Control group & $P$-value \\
\hline Total & 92 & 110 & \\
\hline \multicolumn{4}{|l|}{ Age years } \\
\hline Mean (SD) & $44.9(18.3)$ & $37.5(19.8)$ & 0.007 \\
\hline Range & $14-83$ & $1-84$ & \\
\hline \multicolumn{4}{|l|}{ Gender (n) } \\
\hline Male & 44 & 51 & 0.836 \\
\hline Female & 48 & 59 & \\
\hline \multicolumn{4}{|l|}{ Presenting VA (LogMAR) } \\
\hline Mean VA LogMAR (SD) & $1.8(0.5)$ & I.3 (0.7) & $<0.001$ \\
\hline VA worse than I.3 LogMAR, n (\%) & $78 / 92$ & $56 / 110$ & $<0.001$ \\
\hline Mean IOP mmHg (SD) & $31.1(7.9)$ & I5.I (5.9) & $<0.001$ \\
\hline \multicolumn{4}{|l|}{ Corneal lesion, n (\%) } \\
\hline Severe keratitis & $40(43.5)$ & $88(80)$ & $<0.001$ \\
\hline Moderate keratitis & $52(56.5)$ & $22(20)$ & \\
\hline \multicolumn{4}{|l|}{ Anterior chamber cells reaction, $\mathrm{n}(\%)$} \\
\hline Mild to moderate reaction & $17(18.5)$ & $55(50)$ & $<0.001$ \\
\hline Severe reaction & $75(81.5)$ & $55(50)$ & \\
\hline \multicolumn{4}{|l|}{ Identified organisms (\%) } \\
\hline Bacteria & 45.6 & 66.4 & 0.06 \\
\hline Fungi & 34.8 & 21.8 & \\
\hline Acanthamoeba & 2.2 & 1.8 & \\
\hline Mixed & 4.3 & 1.8 & \\
\hline Unidentified & 13.1 & 8.2 & \\
\hline \multicolumn{4}{|l|}{ Underlying ophthalmic disease } \\
\hline Contact lens related & 18 & 36 & 0.04 \\
\hline Lids and ocular surface abnormalities & I & 8 & 0.28 \\
\hline Previous cataract surgery & 10 & 5 & 0.09 \\
\hline \multicolumn{4}{|l|}{ Underlying systemic disease } \\
\hline Diabetes mellitus & 9 & 5 & 0.14 \\
\hline Hypertension & 8 & 9 & 0.89 \\
\hline Anti-HIV positive & 4 & 2 & 0.41 \\
\hline Smoking & 21 & 14 & 0.06 \\
\hline Alcohol & 16 & 13 & 0.26 \\
\hline
\end{tabular}

Abbreviations: OHT, ocular hypertension; VA, visual acuity; IOP, intraocular pressure. 
Table 2 Pathogenic organisms isolated from culture or corneal biopsy

\begin{tabular}{|c|c|}
\hline Organism & $\mathbf{n}$ \\
\hline \multicolumn{2}{|l|}{ Bacteria } \\
\hline Pseudomonas aeruginosa & 38 \\
\hline Mycobacterium abscessus & 4 \\
\hline MSSA Staphylococcus aureus & 3 \\
\hline Serratia marcescens & 3 \\
\hline Aeromonas hydrophila & 2 \\
\hline Gram-negative bacilli & 2 \\
\hline Gram-positive cocci & 1 \\
\hline Staphylococcus (coagulase negative) & 1 \\
\hline Streptococcus pneumoniae & 1 \\
\hline Beta-hemolytic streptococcus (not group D) & 1 \\
\hline Stenotrophomonas maltophilia & 1 \\
\hline Pseudomonas mirabilis & 1 \\
\hline Enterobacter cloacae & I \\
\hline Enterobacter aerogenes & 1 \\
\hline Klebsiella pneumoniae & 1 \\
\hline Moraxella spp. & 1 \\
\hline \multicolumn{2}{|l|}{ Fungi } \\
\hline Fusarium spp. & 9 \\
\hline Aspergillus spp. & 8 \\
\hline Dematiaceous mold & 2 \\
\hline Candida spp. & 1 \\
\hline Mucor spp. & 1 \\
\hline Pythium spp. & 1 \\
\hline Acanthamoeba & 3 \\
\hline
\end{tabular}

between IOP increase and age, gender, presenting VA, size of ulcer, AC cells reaction, types of pathogenic organisms, and underlying ophthalmic and systemic diseases. In the multivariate analysis, we included any variable having a significant univariate $P$-value of $<0.1$. We found that large and deep keratitis and severe cells' reaction in AC were associated with the risk of IOP elevation during the course of disease, whereas age, severe vision loss on presentation, types of pathogenic organisms, contact lens-related keratitis, previous cataract surgery, and smoking were statistically significant predictors in simple logistic regression, but not in multiple logistic regression. Other underlying ocular and systemic diseases were not associated with IOP elevation in both simple and multiple logistic regression analyses (Table 4).

Subgroup analyses of patients with secondary OHT demonstrated that, severity of keratitis, magnitude of IOP elevation, medical and surgical treatment to control IOP, and length of stay were significantly different between bacterial and fungal keratitis (Table 5). Fungal keratitis with IOP elevation was associated with more severe keratitis (OR 3.8, 95\% CI 1.4-10.1) and more severe AC cells' reaction (OR 4.69, 95\% CI 0.95-23.2). Magnitude of IOP increase was significantly higher in fungal keratitis compared to bacterial keratitis patients $(P=0.032)$. Number of glaucoma medications used and rates of laser cyclophotocoagulation procedure required to control IOP were also significantly higher in fungal infection $(P=0.004$ and $P=0.001$, respectively). A total of nine patients with elevated IOP required enucleation procedure due to uncontrolled endophthalmitis. Fungal organisms were the cause in seven patients and the organism was not identified in two patients. None of the patients with bacterial, acanthamoebic, and mixed organism keratitis as well as fungal keratitis without IOP elevation had undergone enucleation during active infection.

Long-term follow-up data were available in 69 from 92 patients with elevated IOP during active infection. Of all 23 patients whose records were unavailable, 14 were referred back to their primary ophthalmologists, 8 failed to return for follow-up, and 1 refused treatment, while still taking antiglaucoma medications. The overall median time of follow-up was 11 months (1-142). Long-term data of IOP measurement with Tono-pen XL and/or Goldmann applanation tonometer, when suitable, were included for analysis. At the last follow-up, 32.9\% (22/69) required long-term antiglaucoma medications to control IOP. The mean IOP was $11.2( \pm 4.5) \mathrm{mmHg}$ in patients without long-term IOP

Table 3 Incidence of secondary OHT in each group of pathogenic organism

\begin{tabular}{|c|c|c|c|c|c|c|}
\hline & $\mathbf{n}$ & Bacteria & Fungi & Acanthamoeba & Mixed & Unidentified \\
\hline \multicolumn{7}{|l|}{ Severe infection (142) } \\
\hline OHT & 79 & 34 & 30 & I & 4 & 10 \\
\hline Non-OHT & 63 & 47 & 10 & 0 & 2 & 4 \\
\hline \multicolumn{7}{|l|}{ Moderate infection (60) } \\
\hline OHT & 13 & 8 & 2 & 1 & 0 & 2 \\
\hline Non-OHT & 47 & 26 & 14 & 2 & 0 & 5 \\
\hline \multicolumn{7}{|l|}{ Organism identification } \\
\hline Culture or corneal biopsy & 86 & 55 & 22 & 3 & 6 & 0 \\
\hline Clinical identification & 116 & 60 & 34 & I & 0 & 21 \\
\hline Total & 202 & 115 & 56 & 4 & 6 & 21 \\
\hline
\end{tabular}

Abbreviation: OHT, ocular hypertension. 
Table 4 Multiple logistic regression analysis of the risk factors associated with IOP elevation

\begin{tabular}{|c|c|c|c|}
\hline Risk factors & Adjusted odds ratio & $95 \% \mathrm{Cl}$ & P-value \\
\hline Age & 1.00 & $0.99-1.03$ & 0.62 \\
\hline Presenting LogMAR VA worse than I.3 & 2.33 & $0.99-5.46$ & 0.05 \\
\hline Severe keratitis & 2.97 & $1.46-6.07$ & 0.003 \\
\hline Severe AC cells' reaction & 2.66 & $1.18-6.00$ & 0.018 \\
\hline Contact lens related & 0.68 & $0.28-1.69$ & 0.41 \\
\hline Previous cataract surgery & 2.81 & $0.73-10.8$ & 0.13 \\
\hline \multicolumn{4}{|l|}{ Causative organism compared to bacteria } \\
\hline Fungus & 1.56 & $0.68-3.62$ & 0.29 \\
\hline Acanthamoeba & 6.25 & $0.57-69.13$ & 0.14 \\
\hline Mixed & 1.50 & $0.23-9.63$ & 0.67 \\
\hline Unidentified & 2.01 & $0.6 I-6.64$ & 0.25 \\
\hline
\end{tabular}

Abbreviations: AC, anterior chamber; IOP, intraocular pressure; VA, visual acuity.

elevation and $20.2( \pm 16.7) \mathrm{mmHg}$ in patients with persistent IOP elevation $(P<0.001)$. Mean number of medications at the last follow-up was $1.59( \pm 0.7)$. Final presenting VA was $0.78( \pm 0.83)$ LogMAR as compared to $0.70( \pm 0.63)$ LogMAR in patients with and without persistent IOP elevation, respectively $(P=0.65)$. The long-term clinical data separated by causative organism are presented in Table 6. Patients with fungal keratitis seemed to have an increased risk of developing persistent IOP elevation and blindness (VA worse than 1.3 LogMAR) at the last follow-up. Nevertheless, in multivariate analysis, the risk factors associated with persistent elevated IOP were age (OR 1.06, 95\% CI 1.02-1.10) and hyperosmotic agents used during active keratitis (OR 5.4, 95\% CI 1.20-23.87), with the $P$-values 0.007 and 0.028 , respectively. For final visual outcome, only age was a significant risk factor for blindness (OR 1.08, 95\% CI 1.03-1.13; $P=0.002$ ) among the patients with secondary OHT.

\section{Discussion}

In this study, we evaluated the incidence of and the risk factors for secondary OHT in moderate to severe infectious ulcerative keratitis at a tertiary care center. We found a high incidence rate $(45.5 \%)$ of IOP elevation developed in our patients. Previous studies reported the incidence of OHT associated with active microbial keratitis to be $28 \%-49 \%{ }^{1,4,5}$ One possible explanation for the differences in the incidence rates may be the different severity of keratitis and underlying ocular diseases among studies. We excluded the patients who had glaucoma or OHT in either the study eyes or the fellow eyes, in order to evaluate the effect of infectious keratitis on IOP. There is a possibility that some patients might have had unilateral OHT or glaucoma in the affected eye, but the number of such patients is considered to be small. Moreover, the association between OHT and underlying glaucoma was not found in a previous study that did not exclude the patients

Table 5 Comparison between bacterial and fungal keratitis with elevated IOP

\begin{tabular}{|c|c|c|c|c|}
\hline & \multicolumn{2}{|c|}{ Pathogenic organisms } & \multirow[t]{2}{*}{ P-value } & \multirow[t]{2}{*}{ OR (95\% CI) } \\
\hline & Bacterial & Fungal & & \\
\hline Number & 42 & 32 & & \\
\hline Mean age, years (SD) & $37.8(19.1)$ & $51.8(14.5)$ & $<0.001$ & \\
\hline Presenting VA worse than I.3 LogMAR, n (\%) & $35(83.3)$ & $28(87.5)$ & 0.618 & $1.4(0.4-5.3)$ \\
\hline Mean IOP, mmHg (SD) & $28.7(6.5)$ & $34.8(8.7)$ & $<0.001$ & \\
\hline Severe keratitis, n (\%) & $17(40.5)$ & 23 (7I.9) & 0.007 & $3.8(1.4-10.1)$ \\
\hline Severe AC cells' reaction, $n(\%)$ & $32(76.2)$ & $30(93.8)$ & 0.042 & $4.69(0.95-23.2)$ \\
\hline No of antiglaucoma medications (n) & & & 0.004 & \\
\hline $0-2$ medications & 13 & 6 & & \\
\hline 3-4 medications & 29 & 20 & & \\
\hline 5 medications & 0 & 6 & & \\
\hline Hyperosmotic agents required, n (\%) & $16(38.1)$ & $22(68.8)$ & 0.009 & $3.6(1.4-9.5)$ \\
\hline Laser cyclophotocoagulation required, n (\%) & I (2.4) & $9(28.1)$ & $<0.001$ & $16.0(1.9-134.8)$ \\
\hline Surgery to control infection, n (\%) & 0 & $15(46.9)$ & $<0.001$ & \\
\hline Therapeutic keratoplasty or scleral graft & 0 & 8 & & \\
\hline Enucleation & 0 & 7 & & \\
\hline Length of stay, days (SD) & $22.2(15.4)$ & $35.3(23.5)$ & 0.009 & \\
\hline
\end{tabular}

Abbreviations: AC, anterior chamber; IOP, intraocular pressure; VA, visual acuity. 
Table 6 Long-term clinical outcome in patients with secondary ocular hypertension

\begin{tabular}{|c|c|c|c|c|c|}
\hline & Bacteria & Fungi & Acanthamoeba & Mixed organism & Unidentified \\
\hline Number & 34 & 20 & 2 & 4 & 9 \\
\hline \multicolumn{6}{|l|}{ F/U duration (months) } \\
\hline Median (range) & $4.23(1-112)$ & $13(1-134)$ & $5.1(3-6)$ & $10.7(8-65)$ & $23.2(2-142)$ \\
\hline Final VA $>1.3$ LogMAR & $17.6 \%(6 / 34)$ & $60 \%(12 / 20)$ & $50 \%(1 / 2)$ & $25 \%(1 / 4)$ & $33.3 \%(3 / 9)$ \\
\hline \multicolumn{6}{|l|}{ Final IOP, mmHg } \\
\hline Mean (SD) & $13.9(6.5)$ & I5.4 (I7.5) & $10(2)$ & II.5 (4.4) & $12(1 \mid .8)$ \\
\hline Median & 12 & 14 & 10 & 11.5 & 12 \\
\hline Glaucoma medications used at the last follow-up & II.8\% (4/34) & $55 \%(11 / 20)$ & $50 \%(1 / 2)$ & $50 \%(2 / 4)$ & $44.4 \%(4 / 9)$ \\
\hline \multicolumn{6}{|l|}{ Glaucoma surgery (n) } \\
\hline Laser cyclophotocoagulation & 1 & 8 & & 1 & 2 \\
\hline Trabeculectomy & & 2 & & & I \\
\hline Glaucoma drainage device & & I & & & - \\
\hline Final VA $<0.48$ LogMAR & $41.2 \%(14 / 34)$ & $5 \%(1 / 20)$ & $50 \%(1 / 2)$ & $25 \%(1 / 4)$ & $11.1 \%(1 / 9)$ \\
\hline Final VA > $>1.3$ LogMAR & $17.6 \%(6 / 34)$ & $60 \%(12 / 20)$ & $50 \%(1 / 2)$ & $25 \%(1 / 4)$ & $33.3 \%(3 / 9)$ \\
\hline
\end{tabular}

Abbreviations: F/U, follow-up; IOP, intraocular pressure; VA, visual acuity.

with known underlying glaucoma. ${ }^{1}$ We also excluded patients who presented with corneal perforation, which will profoundly affect the results, from the study. We were not able to measure the IOP in some patients initially due to extensive corneal involvement. The antiglaucoma medications might be prescribed based on digital palpation compared to the other eye. If the IOP measured was higher than $21 \mathrm{mmHg}$ or consistently $\geq 8 \mathrm{mmHg}$ higher than the other eye at any time before the resolution of keratitis, the patients were included in the OHT group and the maximal IOP recorded was used in analysis.

Our current data revealed that size and depth of corneal involvement were significantly associated with the risk of IOP elevation, as previously shown by Zarei-Ghanavati et al. ${ }^{1}$ Even though we excluded small-sized ulcer $(<2 \mathrm{~mm}$ in diameter) from the study, severe keratitis is still the major risk of OHT development compared to moderate keratitis (OR 2.97, 95\% CI 1.49-6.07; $P=0.003$ ). The other factor associated with secondary OHT was more severe AC cells' reaction, which also represented more severe infections (OR $2.66,95 \%$ CI $1.18-6.00 ; P=0.018)$. We did not find the association between diabetes mellitus and IOP elevation, as was previously demonstrated in Zarei-Ghanavati et al's paper. Also, in contrast to that paper, history of previous ocular surgery was not related to IOP elevation. The pathophysiology of IOP elevation in infectious keratitis is believed to be multifactorial. Trabecular meshwork may be blocked by inflammatory cells, plasma protein, and toxic products from the damaged cornea, or closed by contraction of the inflammatory membrane in the aqueous drainage angle. We excluded previous ocular surgeries other than uncomplicated cataract surgery from the study. Therefore, our patients who underwent previous ocular surgeries were more likely to have underlying non-compromised aqueous drainage angles than those in the previous study. In addition, the younger age group of the patients in this study $(46.13[ \pm 20.41]$ vs 55.44 $[ \pm 22.86]$ years) could be responsible for the different risk analysis results.

One-third (32.9\%) of the patients with OHT had persistent elevated IOP that required long-term antiglaucoma medications after keratitis was cured. Although chronically sustained elevated IOP can lead to glaucoma development undoubtedly, anatomical and functional glaucomatous damage is difficult to monitor due to corneal scar. At the last follow-up visit, the IOP was controlled with one to two medications in most of the cases. No statistical difference in severe visual impairment outcome was observed between the eyes with and without persistent elevated IOP. Mean presenting VA was LogMAR $0.78(20 / 120)$ in eyes with chronic persistent elevated IOP vs LogMAR $0.70(20 / 100)$ in eyes without persistent elevated IOP. Older age was found to be a significant risk factor for persistent IOP elevation and poor visual outcome after keratitis resolved. These findings may suggest that age-related changes in immune responses increase morbidity from infectious keratitis in older patients. Furthermore, previous studies have shown that, as age increases, trabecular cells decline at a similar rate with corneal endothelial cell loss. ${ }^{6-8}$ Trabecular cells have a role in maintaining the trabecular meshwork function, while corneal endothelial cells maintain the relatively dehydrated state of cornea. Infectious keratitis with AC inflammation in older patients can cause more permanent damage to both the cornea and the trabecular meshwork, thus resulting in more persistent IOP elevation and poorer visual outcome at the last follow-up. Additionally, the reason for optic atrophy in patients with severe ocular inflammation might not be entirely 
elevated IOP. It may result from vascular insufficiency at the optic nerve head, which also varies with increasing age. Another significant risk factor for persistent elevated IOP is hyperosmotic agents used during active keratitis. The requirement of hyperosmotic agents to control IOP reflects the more severe OHT, which thereafter becomes the risk factor for persistent IOP elevation.

Since Thailand is a tropical and agricultural country, we observed higher incidences of fungal infections compared to Zarei-Ghanavati et al's study (27.7\% vs $13.6 \%)$. We observed higher chance to receive surgical procedure to control infection during active keratitis as well as glaucoma procedure after infection was cured in patients with fungal keratitis. However, an association between the types of pathogenic organisms and OHT was not found to be statistically significant in either study. Likewise, long-term data analysis did not show an association between the types of pathogenic organisms and chronic persistent IOP elevation. The higher rates of surgical procedures performed in fungal keratitis could have resulted from different clinical characteristics of keratitis and secondary glaucoma from different types of organisms. In addition to the pathophysiology of IOP elevation discussed previously, fungal keratitis has been reported to be complicated by malignant glaucoma due to pupillary block from lens-iris-exudate apposition causing uniform shallowing of the AC. ${ }^{9,10}$ A malignant glaucoma secondary to fungal keratitis can lead to rapid progression of glaucomatous damage or corneal perforation. Treatment with therapeutic keratoplasty, lens removal, and posterior chamber wash with antifungal was recommended. ${ }^{9-11}$ Overall, 7 out of 202 eyes, all in fungal keratitis with persistent elevated IOP group, were complicated by corneal perforation that required scleral graft and diode laser cyclophotocoagulation. At the last follow-up, three of seven eyes developed phthisis bulbi. It is inconclusive whether fungal infection, severity of infection, corneal perforation and therapeutic keratoplasty, diode laser cyclophotocoagulation procedure, or a combination of multiple conditions is the major risk factor for atrophic disorganized globe. Further prospective study to evaluate the characteristics of IOP elevation and long-term outcome is beneficial.

Our current study has several limitations. First, due to its retrospective design, we were unable to obtain the history of self-medication with topical steroids, duration of symptoms before initial examination, and clinical signs to identify the mechanism of elevated IOP. Second, IOP measurement during the course of diseases can be inaccurate or inconsistent due to surface irregularities and alteration in the biomechanical properties of the cornea.
Lastly, since our hospital is a tertiary referral center, most of the patients had been transferred from other hospitals for non-resolving keratitis. The patients in our study may have more severe diseases, limiting the generalizability of the results. Also, when the infection was under control, during tapering off of the medications, we referred some of the patients back to their primary ophthalmologists, especially those without complications such as elevated IOP or corneal perforation. The long-term data of these patients were not available.

\section{Conclusion}

We found a high incidence rate of elevated IOP in moderate or severe infectious ulcerative keratitis. Severe degrees of corneal infiltration and severe intraocular inflammation were associated with secondary OHT. One-third of the patients with OHT developed persistent elevated IOP after keratitis was healed. Older age and hyperosmotic agents used during active keratitis were significantly associated with persistent elevated IOP. However, most of the eyes could be controlled with one to two medications. Persistent elevated IOP after infectious keratitis resolved was not associated with poorer visual outcome.

\section{Disclosure}

The authors report no conflicts of interest in this work.

\section{References}

1. Zarei-Ghanavati S, Baghdasaryan E, Ramirez-Miranda A, et al. Elevated intraocular pressure is a common complication during active microbial keratitis. Am J Ophthalmol. 2011;152(4):575.e1-581.e1.

2. Jones DB. Decision-making in the management of microbial keratitis Ophthalmology. 1981;88(8):814-820.

3. Jabs DA, Nussenblatt RB, Rosenbaum JT; Standardization of Uveitis Nomenclature (SUN) Working Group. Standardization of uveitis nomenclature for reporting clinical data. Results of the First International Workshop. Am J Ophthalmol. 2005;140(3):509-516.

4. Hongyok T, Leelaprute W. Corneal ulcer leading to evisceration or enucleation in a tertiary eye care center in Thailand: clinical and microbiological characteristics. JMed Assoc Thai. 2016;99(Supp1 2):S116-S122.

5. Constantinou M, Jhanji V, Tao LW, Vajpayee RB. Clinical review of corneal ulcers resulting in evisceration and enucleation in elderly population. Graefes Arch Clin Exp Ophthalmol. 2009;247(10):1389-1393.

6. Alvarado J, Murphy C, Polansky J, Juster R. Age-related changes in trabecular meshwork cellularity. Invest Ophthalmol Vis Sci. 1981;21(5): 714-727.

7. Laule A, Cable MK, Hoffman CE, Hanna C. Endothelial cell population changes of human cornea during life. Arch Ophthalmol. 1978; 96(11):2031-2035.

8. Blatt HL, Rao GN, Aquavella JV. Endothelial cell density in relation to morphology. Invest Ophthalmol Vis Sci. 1979;18(8):856-859.

9. Kuriakose T, Thomas PA. Keratomycotic malignant glaucoma. Indian J Ophthalmol. 1991;39(3):118-121.

10. Chin GN, Goodman NL. Aspergillus flavus keratitis. Ann Ophthalmol. 1978;10(4):415-418.

11. Jones BR, Jones DB, Richards AB. Surgery in the management of keratomycosis. Trans Ophthalmol Soc U K. 1970;89:887-897. 


\section{Publish your work in this journal}

Clinical Ophthalmology is an international, peer-reviewed journal covering all subspecialties within ophthalmology. Key topics include: Optometry; Visual science; Pharmacology and drug therapy in eye diseases; Basic Sciences; Primary and Secondary eye care; Patient Safety and Quality of Care Improvements. This journal is indexed on

PubMed Central and CAS, and is the official journal of The Society of Clinical Ophthalmology (SCO). The manuscript management system is completely online and includes a very quick and fair peer-review system, which is all easy to use. Visit http://www.dovepress.com/ testimonials.php to read real quotes from published authors. 